\title{
Effect of Mild Hypothermia and Hypoxia on Blood Flow and Oxygen Consumption of the Fetal Sheep Brain
}

\author{
HIROMITSU CHIHARA, ARLIN B. BLOOD, CHRISTIAN J. HUNTER, AND GORDON G. POWER \\ Loma Linda University School of Medicine, Center for Perinatal Biology, Loma Linda, California 92354, \\ U.S.A.
}

\begin{abstract}
This study was undertaken to measure the effects of mild hypothermia on cerebral blood flow and metabolism and cardiovascular responses to hypoxia in the fetal sheep. Near-term fetal sheep were chronically instrumented with laser Doppler flowmetry in the parietal cortex for measurement of relative changes in cerebral blood flow, as well as with arterial and sagittal sinus catheters for measurement of oxygen extraction by the brain and a cooling coil around the fetal thorax. Fetuses were studied during cooling alone, cooling with superimposed maternal hypoxia to achieve a fetal arterial $\mathrm{Po}_{2}$ of 1.33 to $1.60 \mathrm{kPa}$, or hypoxia alone. In response to cooling alone $\left[1.6^{\circ} \pm 0.1^{\circ} \mathrm{C}\right.$ (mean $\left.\pm \mathrm{SEM}\right)$ decrease in brain temperature], fetal blood pressure and heart rate both increased significantly whereas cerebral blood flow decreased $14 \pm 4 \%$, commensurate with a $24 \pm 8 \%$ decline in cerebral metabolic rate. Administration of moderate hypoxia during cooling resulted in a significant increase in cerebral blood flow, decreased heart rate, and no further increase in blood
\end{abstract}

\section{ABSTRACT}

pressure. In response to hypoxia alone, fetal blood pressure was significantly increased, heart rate was decreased, and cerebral blood flow increased by $24 \pm 8 \%$, whereas cerebral metabolic rate decreased by $38 \pm 13 \%$. Arteriovenous oxygen extraction was unchanged by cooling alone but increased significantly in response to hypoxia administered during cooling. We therefore conclude that oxygen delivery to the fetal sheep brain remains coupled to metabolic rate during hypothermia and that hypothermia does not impair the compensatory cardiovascular responses of the fetus to acute moderate hypoxia. (Pediatr Res 54: 665671, 2003)

\section{OD, outer diameter \\ Abbreviations \\ $\mathbf{Q}_{10}$ coefficient, ratio of two different rates of oxygen consumption at two temperatures separated by $10^{\circ} \mathrm{C}$}

Under normal physiologic conditions, the rate at which the brain is consuming oxygen is the primary determinant of cerebral oxygen delivery. In the newborn and adult, this relationship has been demonstrated by observing that hypothermia or barbiturate-induced decreases in cerebral oxygen consumption result in comparable decreases in cerebral oxygen delivery (1-6). Although the relationship between cerebral oxygen delivery and consumption has been studied extensively in the fetus by inducing changes in arterial oxygen content (7), the approach of altering cerebral oxygen consumption and observing changes in oxygen delivery has not been fully explored. Because of the large difference in oxygen tensions between the fetus and adult (approximately $3.33 \mathrm{kPa}$ versus approximately

Received November 12, 2002; accepted April 23, 2003.

Correspondence: Gordon G. Power, M.D., Center for Perinatal Biology, Loma Linda University School of Medicine, 11234 Anderson Street, Loma Linda, CA 92354, U.S.A.; e-mail: gpower@som.llu.edu

Supported in part by a grant from the U.S. Public Health Service, National Institutes of Health (HL 65494).

H.C. and A.B.B. shared equally in this work.

DOI: 10.1203/01.PDR.0000084115.31416.17
$12.67 \mathrm{kPa}$ ), the finding that decreases in cerebral oxygen consumption result in decreased cerebral oxygen delivery in the newborn and adult cannot be assumed for the fetus.

Cerebral hypothermia has significant neuroprotective properties when administered to fetal sheep in conjunction with or after hypoxic-ischemic insult $(8,9)$ and is being evaluated for its clinical therapeutic benefit (10). Under normoxic conditions, cerebral hypothermia results in decreases in cerebral oxygen consumption and parallel reductions of cerebral blood flow and oxygen delivery (4). These responses are associated with increased cerebrovascular resistance, but little or no change in cerebral oxygen extraction. In contrast, moderate hypoxic stress results in increased cerebral blood flow and oxygen extraction, and decreased cerebrovascular resistance (7). Whether these protective mechanisms during hypoxia remain intact during hypothermia has not been studied and is of some concern in the consideration of the safety of hypothermia as a therapeutic treatment after hypoxic-ischemic insult.

The present study was designed to test the hypothesis that fetal hypothermia results in decreased cerebral oxygen consumption that is accompanied by a proportionate reduction in 
cerebral blood flow and cerebral oxygen delivery. A second purpose was to test the hypothesis that the normal fetal cardiovascular responses to hypoxic stress remain intact during hypothermia. The approach used was to reduce the brain temperature of chronically instrumented fetal sheep while measuring arterial blood pressure, heart rate, oxygen delivery by laser Doppler blood flow measurement, and cerebral oxygen use by the Fick principle with and without superimposed systemic hypoxia.

\section{METHODS}

Experimental preparation. The studies were approved by the Animal Use Committee of Loma Linda University. Timedated pregnant, mixed-breed Western ewes were obtained from a local supplier (Nebeker Ranch, Lancaster, CA, U.S.A.). Fourteen pregnant ewes at $126 \pm 2 \mathrm{~d}$ gestational age were used (term $=147 \mathrm{~d})$. The ewes were fasted for $18 \mathrm{~h}$ before surgery, but were otherwise allowed free access to compressed hay pellets and water. Anesthesia was induced with thiopental (10 $\mathrm{mg} / \mathrm{kg}$, i.v.) and was maintained by administration of $1.5-2.5 \%$ halothane in oxygen through a cuffed endotracheal tube. Under sterile conditions, a temperature probe (type 402; YSI, Yellow Springs, OH, U.S.A.) was placed in the maternal descending aorta through the right femoral artery to measure the maternal core temperature. A maternal femoral venous catheter was inserted to administer fluids ( $1 \mathrm{~L}$ of $0.9 \% \mathrm{NaCl}$ ) during surgery. A midline abdominal incision was made in the maternal skin and uterine wall, and the fetal head and upper torso were partially delivered. A 5-m coil of plastic tubing was placed around the fetal thorax for cooling as reported previously (11). A thermocouple (type IT-18; Physitemp, Clifton, NJ, U.S.A.) was inserted into the right brachial artery with the tip advanced to the ascending aorta to measure the fetal core body temperature. A polyvinyl catheter (1.5 mm OD) was inserted into each lingual artery for withdrawal of blood samples and measurement of fetal arterial blood pressure.

Incisions were made in the fetal scalp, and the skull was exposed. Sagittal sinus centesis was performed $5 \mathrm{~mm}$ anterior to bregma, and a polyvinyl catheter $(1.8 \mathrm{~mm}$ OD) was inserted $10 \mathrm{~mm}$ for blood sampling. For measurement of cerebral perfusion, laser Doppler flowmetry probes (type LDF100A; Biopac Systems, Santa Barbara, CA, U.S.A.) were inserted to a depth of $5 \mathrm{~mm}$ below the dura into the parasagittal parietal cortex through $0.5-\mathrm{mm}$ burr holes drilled $5 \mathrm{~mm}$ lateral to the midline and $20 \mathrm{~mm}$ posterior to the coronal suture. Details of this method have been described previously (12). Thermocouples (type IT-23; Physitemp), for measurement of brain temperature, were inserted to the same depth through burr holes in the cerebral cortex $15 \mathrm{~mm}$ anterior to the laser Doppler flow probe. The catheter and probes were secured to the skull with rubber dam and tissue glue. An additional temperature probe (type 402; YSI) and catheter (2.2 $\mathrm{mm} \mathrm{OD}$ ) were placed on the tip of the fetal nose to measure amniotic fluid temperature to instill antibiotics into the amniotic cavity. The scalp and uterine incisions were sutured closed. All probes, vascular catheters, and plastic tubing were exteriorized through the maternal flank and protected in a nylon pouch attached to the maternal skin. The ewes were allowed to recover from surgery for at least $96 \mathrm{~h}$ before study. During the recovery period, penicillin G $4 \times 10^{4} \mathrm{U}$ was administered intramuscularly to the ewes once daily, and ampicillin $(500 \mathrm{mg})$ and gentamicin $(80 \mathrm{mg})$ were instilled into the amniotic cavity daily. Cefotaxime (50 mg b.i.d.) was administered i.v. to the fetus twice daily.

Experimental procedure. Each fetus was exposed to three different experimental protocols designated as cooling control, cooling with superimposed hypoxia, and hypoxia control (Fig. 1). The protocols were carried out in the order listed above and were separated by $72 \mathrm{~h}$. Arterial pressure was measured from the lingual artery ipsilateral to the laser Doppler flow probe and brain thermocouple, and blood samples were collected from the contralateral lingual artery. To minimize cerebral temperature disturbances caused by flushing the lingual catheter during blood sample collection, saline flushes containing heparin were warmed to approximately $39.5^{\circ} \mathrm{C}$ before infusion.

Cooling control protocol. The cooling protocol $(n=13)$ consisted of an initial 30-min baseline period followed by a 2-h period of cooling, administered by passing ice water through the cooling coil. The flow rate was adjusted so that the fetal ascending aortic blood temperature decreased by approximately $1.5^{\circ} \mathrm{C}$ to a new stable level during the first hour of cooling. After the cooling period, the fetal status was observed during a 1-h recovery period.

Cooling with superimposed hypoxia protocol. The cooling with hypoxia protocol $(n=13)$ consisted of an initial 30-min baseline period and then a 2-h period of cooling as described above for the cooling control protocol with the addition of a superimposed 30-min period of hypoxia beginning $1 \mathrm{~h}$ after the start of cooling. Hypoxia was administered by having the ewe breathe 10 to $11 \% \mathrm{O}_{2}$ in a balance of $\mathrm{N}_{2}$ passing rapidly (30 $\mathrm{L} / \mathrm{min}$ ) through a plastic bag placed over her head as previously described (12). The hypoxic interval was followed by a 30-min recovery period while cooling was continued and then a $1-\mathrm{h}$ recovery period without cooling.

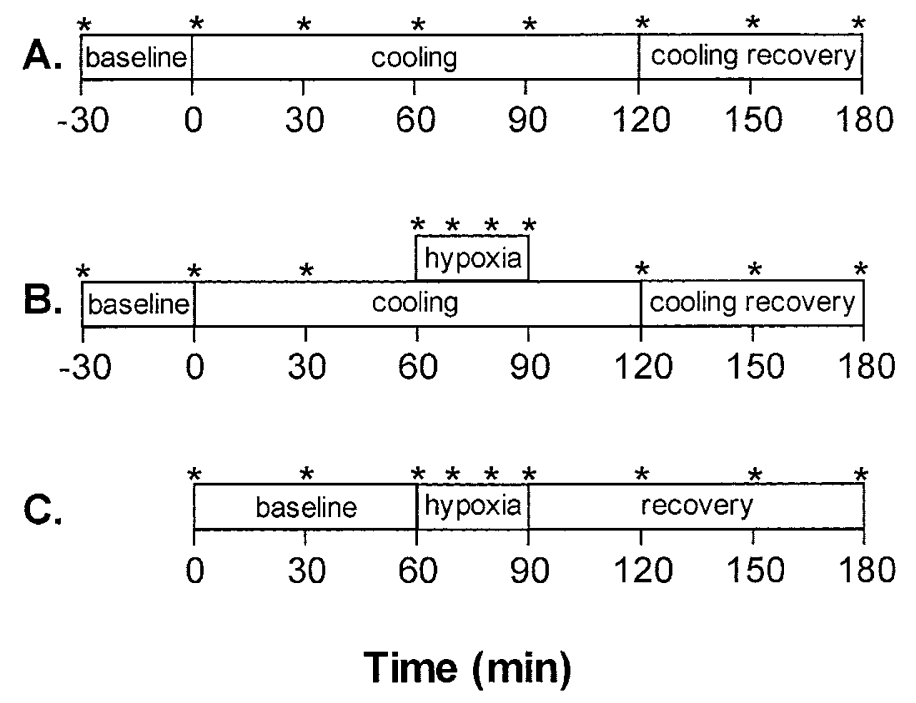

Figure 1. Diagrammatic representation of cooling control $(A)$, cooling with hypoxia $(B)$, and hypoxia control $(C)$ experiments. $\left(^{*}\right.$, blood sampling time points) 
Hypoxia control protocol. The hypoxic control protocol $(n$ $=13$ ) consisted of an initial 1-h baseline followed by a 30-min period of hypoxia, and then 90-min recovery period. All experiments were carried out at room temperature while the ewes stood in a study cage and were performed when the physiologic status of the fetus was stable.

Temperature, cerebral blood flow, and blood pressure measurement. Maternal descending aortic and amniotic fluid temperature were recorded with a resolution of $0.02^{\circ} \mathrm{C}$ (model $4000 \mathrm{~A}$ thermistor; YSI). Fetal body core (ascending aorta) and brain (parasagittal parietal cortex) temperatures were measured using an amplifier-transducer unit capable of linear temperature measurement with a resolution of $0.01^{\circ} \mathrm{C}$. Laser Doppler flowmetry, fetal brain and arterial temperatures, fetal arterial and sagittal sinus blood pressures, amniotic pressure, and heart rate were recorded using an analog to-digital converter system (MP100 System; Biopac Systems) and data acquisition software (Acqknowledge v3.5; Biopac Systems). Thermocouples and temperature probes were calibrated before surgery, and data acquisition equipment was calibrated before each experiment. After euthanasia of the ewe and fetus, probe and catheter positions were verified, and thermocouples were recalibrated to verify that their calibration had remained stable.

Analytical procedures. Fetal arterial and sagittal sinus blood samples (both $0.7 \mathrm{~mL}$ ) were taken at 30-min intervals throughout the experiments. During hypoxic periods, additional blood samples were taken at 10 -min intervals. The first $0.3 \mathrm{~mL}$ of arterial and sagittal sinus blood was analyzed for blood gases and pH (ABL3; Radiometer, Copenhagen, Denmark), oxyhemoglobin saturation, total $\mathrm{Hb}$ concentration, and oxygen content (OSM2 hemoximeter; Radiometer). Fetal arterial temperature was used for calculation of arterial blood gases at each sampling point. The remaining $0.4 \mathrm{~mL}$ of arterial blood was used for measurement of plasma glucose and lactate (model 2700; YSI, Dayton, OH, U.S.A.).

Statistical analysis. Results are expressed as mean \pm SEM. The significance of differences with time was evaluated by one-way ANOVA with repeated measures using Dunnett's post hoc test when the overall $p$ value of the ANOVA was less than 0.05 . Significant differences between cooling with hypoxia experiments and hypoxia control experiments with time were evaluated by two-way ANOVA with repeated measures followed by Bonferroni's post hoc test. The null hypothesis of no effect was rejected at $p<0.05$. Statistical analyses were performed using Graphpad Prism (Graphpad Software Inc., San Diego, CA, U.S.A.).

\section{RESULTS}

Blood sample measurements. Changes in arterial $\mathrm{Hb}$, oxyhemoglobin saturation, $\mathrm{Po}_{2}$, oxygen content, $\mathrm{PCO}_{2}, \mathrm{pH}$, glucose, and lactate levels are presented in Table 1. Data are presented as the mean value of all samples collected for the time period indicated, except for the hypoxia period, in which case only the

Table 1. Responses of arterial blood gases, Hb, oxyhemoglobin saturation, oxygen content, and glucose and lactate to intrauterine cooling and hypoxia

\begin{tabular}{|c|c|c|c|c|c|c|}
\hline & Protocol & Baseline & $\begin{array}{c}0-60 \text { min of } \\
\text { cooling }\end{array}$ & $60-90 \mathrm{~min}$ & $\begin{array}{c}90-120 \mathrm{~min} \\
\text { and hypoxia } \\
\text { recovery }\end{array}$ & $\begin{array}{l}\text { Cooling } \\
\text { recovery }\end{array}$ \\
\hline \multirow[t]{3}{*}{$\mathrm{Hb}(\mathrm{g} / \mathrm{dL})$} & Cooling & $8.5 \pm 0.3$ & $9.3 \pm 0.3^{*}$ & $9.0 \pm 0.4 \dagger$ & $9.0 \pm 0.4$ & $8.4 \pm 0.3$ \\
\hline & Cool + Hyp & $9.7 \pm 0.5$ & $10.2 \pm 0.5$ & $10.3 \pm 0.4 *$ & $9.4 \pm 0.4$ & $8.8 \pm 0.4 \dagger$ \\
\hline & Hypoxia & $9.2 \pm 0.5$ & NA & $9.5 \pm 0.5^{*}$ & $8.9 \pm 0.4$ & NA \\
\hline \multirow[t]{3}{*}{ HbSat (\%) } & Cooling & $58.3 \pm 3.8$ & $49.0 \pm 3.6 \dagger$ & $51.2 \pm 3.7^{*}$ & $48.9 \pm 4.1 \dagger$ & $52.5 \pm 3.0$ \\
\hline & Cool + Hyp & $56.5 \pm 3.6$ & $45.4 \pm 3.2 \dagger$ & $25.5 \pm 2.2 \uparrow 9$ & $46.2 \pm 2.1 \uparrow \S$ & $50.5 \pm 4$ \\
\hline & Hypoxia & $59.9 \pm 1.5$ & NA & $26.2 \pm 2.0 \dagger$ & $58.2 \pm 1.2$ & NA \\
\hline \multirow[t]{3}{*}{$\mathrm{Po}_{2}(\mathrm{kPa})$} & Cooling & $2.60 \pm 0.13$ & $2.20 \pm 0.12 \dagger$ & $2.19 \pm 0.12 \dagger$ & $2.09 \pm 0.12 \dagger$ & $2.44 \pm 0.11$ \\
\hline & Cool + Hyp & $2.68 \pm 0.13$ & $2.10 \pm .15 \dagger$ & $1.28 \pm 0.08+\top$ & $2.09 \pm 0.13 \dagger \S$ & $2.37 \pm 0.13$ \\
\hline & Hypoxia & $2.71 \pm 0.12$ & NA & $1.43 \pm 0.05 \dagger$ & $2.59 \pm 0.12$ & NA \\
\hline \multirow[t]{3}{*}{$\mathrm{O}_{2} \mathrm{ct}(\mathrm{mL} / \mathrm{dL})$} & Cooling & $6.6 \pm 0.4$ & $6.0 \pm 0.3$ & $6.1 \pm 0.4$ & $5.8 \pm 0.4 *$ & $5.9 \pm 0.4$ \\
\hline & Cool + Hyp & $7.4 \pm 0.6$ & $6.1 \pm 0.4^{*}$ & $3.5 \pm 0.3+\Phi$ & $5.8 \pm 0.3 \dagger$ & $6.0 \pm 0.5 \dagger$ \\
\hline & Hypoxia & $7.2 \pm 0.6$ & NA & $3.2 \pm 0.3 \dagger$ & $6.8 \pm 0.5$ & NA \\
\hline \multirow[t]{3}{*}{$\mathrm{PCO}_{2}(\mathrm{kPa})$} & Cooling & $6.44 \pm 0.17$ & $6.32 \pm 0.20$ & $6.17 \pm 0.20$ & $6.25 \pm 0.13$ & $6.52 \pm 0.13$ \\
\hline & Cool + Hyp & $6.71 \pm 0.16$ & $6.52 \pm 0.16$ & $5.57 \pm 0.21 \dagger$ & $6.12 \pm 0.13 \dagger$ & $6.44 \pm 0.08$ \\
\hline & Hypoxia & $6.67 \pm 0.08$ & NA & $5.96 \pm 0.17 \dagger$ & $6.35 \pm 0.12$ & NA \\
\hline \multirow[t]{3}{*}{$\mathrm{pH}$} & Cooling & $7.35 \pm 0.1$ & $7.34 \pm 0.01 *$ & $7.35 \pm 0.01$ & $7.35 \pm 0.01$ & \\
\hline & Cool + Hyp & $7.34 \pm 0.1$ & $7.32 \pm 0.01 *$ & $7.35 \pm 0.2$ & $7.31 \pm 0.01 \dagger \Phi$ & $7.32 \pm 0.01$ \\
\hline & Hypoxia & $7.33 \pm 0.2$ & NA & $7.34 \pm 0.2$ & $7.30 \pm 0.02$ & NA \\
\hline \multirow[t]{3}{*}{ Glucose $(\mathrm{g} / \mathrm{L})$} & Cooling & $17.4 \pm 2.5$ & $23.1 \pm 2.9 \dagger$ & $23.8 \pm 2.9 \dagger$ & $23.9 \pm 3.0 \dagger$ & $21.4 \pm 2.8 \dagger$ \\
\hline & Cool + Hyp & $15.4 \pm 1.9$ & $22.9 \pm 3.0 \dagger$ & $25.0 \pm 2.8 \dagger$ & $25.8 \pm 3.3 \dagger$ & $20.3 \pm 2.5^{*}$ \\
\hline & Hypoxia & $16.7 \pm 2.0$ & NA & $21.3 \pm 1.8 *$ & $20.1 \pm 1.6 \dagger$ & NA \\
\hline \multirow[t]{3}{*}{ Lactate $(\mathrm{mM})$} & Cooling & $1.4 \pm 0.1$ & $2.5 \pm 0.4 \dagger$ & $2.6 \pm 0.4 \uparrow$ & $2.7 \pm 0.5 \dagger$ & $2.5 \pm 0.4 \dagger$ \\
\hline & Cool + Hyp & $1.4 \pm 0.1$ & $2.6 \pm 0.3 *$ & $4.8 \pm 0.5+q$ & $4.8 \pm 0.6 \div q$ & $4.0 \pm 0.6 \dagger$ \\
\hline & Hypoxia & $1.3 \pm 0.1$ & NA & $3.6 \pm 0.5 \dagger$ & $3.5 \pm 0.6 \dagger$ & NA \\
\hline
\end{tabular}

* Significant difference from baseline, $p<0.05$.

$\dagger$ Significant difference from baseline, $p<0.01$.

- Significant difference from cooling control experiments, $p<0.05$.

$\S$ Significant difference from cooling + hypoxia experiments, $p<0.05$.

Abbreviations used: HbSat, $\mathrm{Hb}$ saturation; $\mathrm{O}_{2}$ ct, oxygen saturation; Hyp, hypoxia; NA, not applicable. 
samples collected at the end of the hypoxic period were used. There were no significant differences among the baseline periods of the three protocols for any of the measured variables. Significant differences with time and among the three protocols are noted in Table 1 .

Brain temperature. Changes in fetal brain temperature during each of the protocols are presented in Figure 2. Brain temperatures were the same during the baseline periods in the three protocols, averaging $39.71^{\circ} \pm 0.09^{\circ} \mathrm{C}$. With cooling, brain temperature decreased by an average of $1.6^{\circ} \pm 0.1^{\circ} \mathrm{C}$ to an average of $38.10^{\circ} \pm 0.9^{\circ} \mathrm{C}$ during the second hour. There were no significant differences between the brain temperatures measured with and without superimposed hypoxia.

Cerebral oxygen consumption. Changes in cerebral oxygen consumption are shown in Figure $3 \mathrm{~A}$. As a result of failure to maintain catheter patency or failure of the laser Doppler flow probe, useful data were obtained from seven animals in the cooling control experiments, six animals in the cooling with hypoxia experiments, and seven animals in the hypoxia control experiments. In response to cooling alone, cerebral oxygen consumption declined and became measurably less than baseline after $60 \min (-24 \pm 8 \%)$. In experiments with both cooling and hypoxia, cerebral oxygen consumption declined similarly with cooling $(-23 \pm 4 \%)$ and remained depressed with superimposed administration of hypoxia $(-26 \pm 7 \%)$. Values returned to baseline during recovery periods. In the hypoxia control experiments, cerebral oxygen consumption decreased $38 \pm 13 \%$ during the hypoxic interval and rose significantly above baseline during recovery $(+106 \pm 22 \%)$.

The correlation of cerebral oxygen consumption and oxygen delivery is shown in Figure $3 B$. Data points are for all measurements for the cooling control experiments and for measurements during the baseline period and the first $60 \mathrm{~min}$ of cooling during the cooling with hypoxia protocol. Regression analysis provided a slope of $0.56 \pm 0.06$, significantly different from zero $\left(r^{2}=0.52 ; p<0.05\right)$.

Arterial blood pressure, cerebral blood flow, and cerebrovascular resistance. Changes in fetal cerebral blood flow are shown in Figure $4 A$, arterial blood pressure in Figure $5 A$, and calculated vascular resistance in Figure $4 B$.

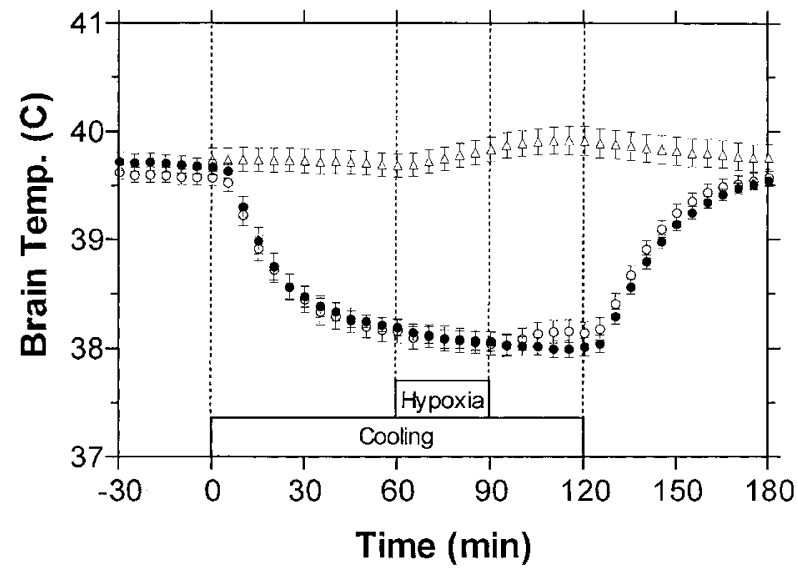

Figure 2. Fetal brain temperatures throughout cooling control $(\bullet, n=13)$, cooling with hypoxia, $(\mathrm{O}, n=11)$, and hypoxia control $(\Delta, n=11)$ experiments.
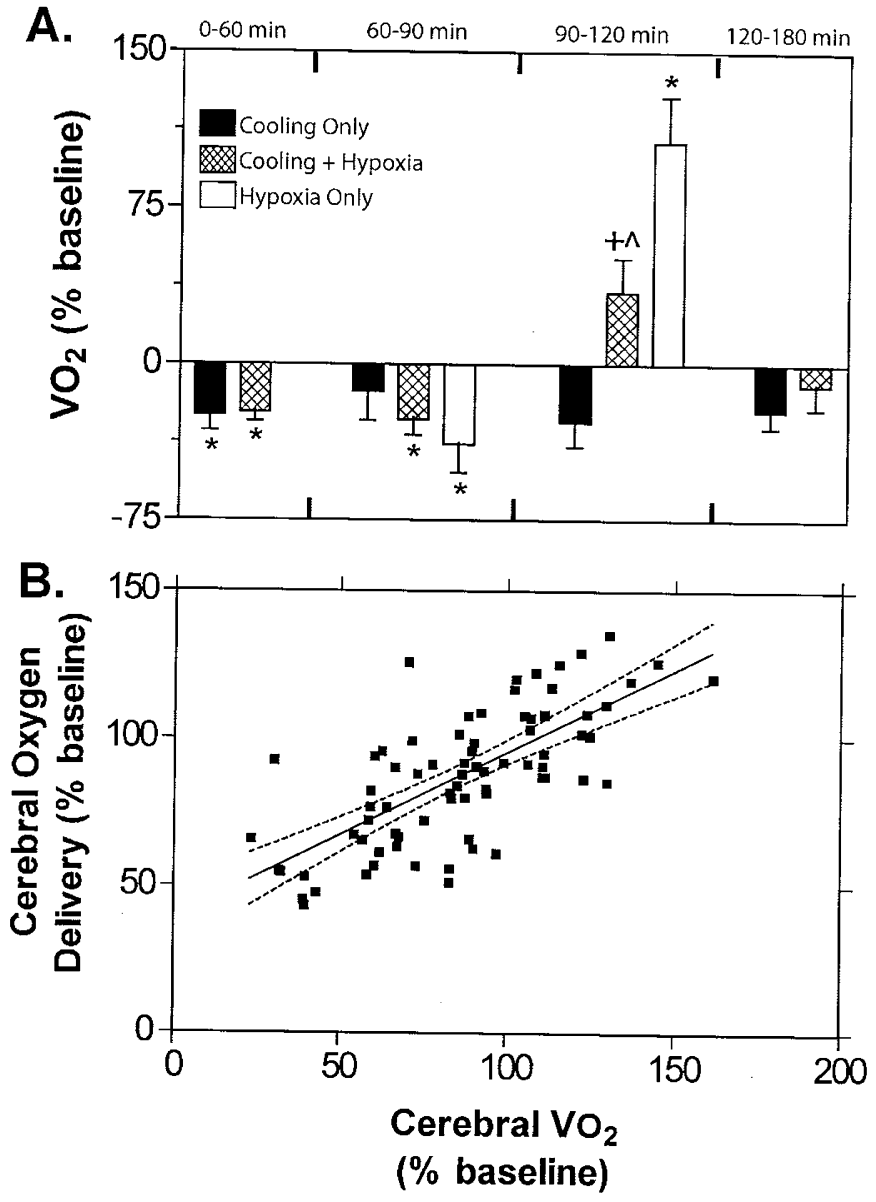

Figure 3. $A$, fetal cerebral oxygen consumption rate $\left(\dot{\mathrm{V}}_{2}\right)$. Data shown under " $0-60 \mathrm{~min}$ " represent the first $60 \mathrm{~min}$ of cooling for cooling control (solid bars, $n=7$ ) and cooling with hypoxia (cross-hatched bars, $n=6$ ) protocols. Data shown under "60-90 min" represent the 60- to 90-min period for the cooling control and cooling with hypoxia protocols, as well as the hypoxia controls (white bars, $n=7$ ). Data shown under "90-120 min" represent the final $30 \mathrm{~min}$ of cooling for the cooling control and cooling with hypoxia protocols, and the 30-min period after hypoxia for the hypoxia controls. Data shown under "120-180 min" represent the 1-h period after discontinuation of cooling in the cooling control and cooling with hypoxia protocols. (*, significant difference from baseline measurements; + , significant difference from the cooling control protocol; $\boldsymbol{\bullet}$, significant difference from the hypoxia control protocol) $B$, X-Y plot of fetal cerebral oxygen consumption $v s$ fetal cerebral oxygen delivery. The line represents a linear regression analysis with a slope of $0.56 \pm 0.06$ (significantly different from 0 ), a $y$ intercept of $32.3 \pm 5.8$, and an $r^{2}=0.51$

In cooling control experiments, blood pressure increased rapidly from a baseline of $42 \pm 1 \mathrm{~mm} \mathrm{Hg}$ on initiation of cooling and then decreased and stabilized at $45 \pm 1 \mathrm{~mm} \mathrm{Hg}$ during the first $90 \mathrm{~min}$ of cooling. Cerebral blood flow decreased significantly to average $14 \pm 4 \%$ below baseline at this time, whereas resistance to blood flow increased by $37 \pm 10 \%$. After termination of cooling, blood pressure and calculated resistance to flow returned to baseline levels, whereas flow remained significantly elevated $13 \pm 3 \%$ above baseline.

In those experiments in which hypoxia was administered in addition to cooling, blood pressure increased rapidly at first on initiation of cooling, as it had in the cooling control experiments, and then stabilized to average $52 \pm 1 \mathrm{~mm} \mathrm{Hg}$ during the 


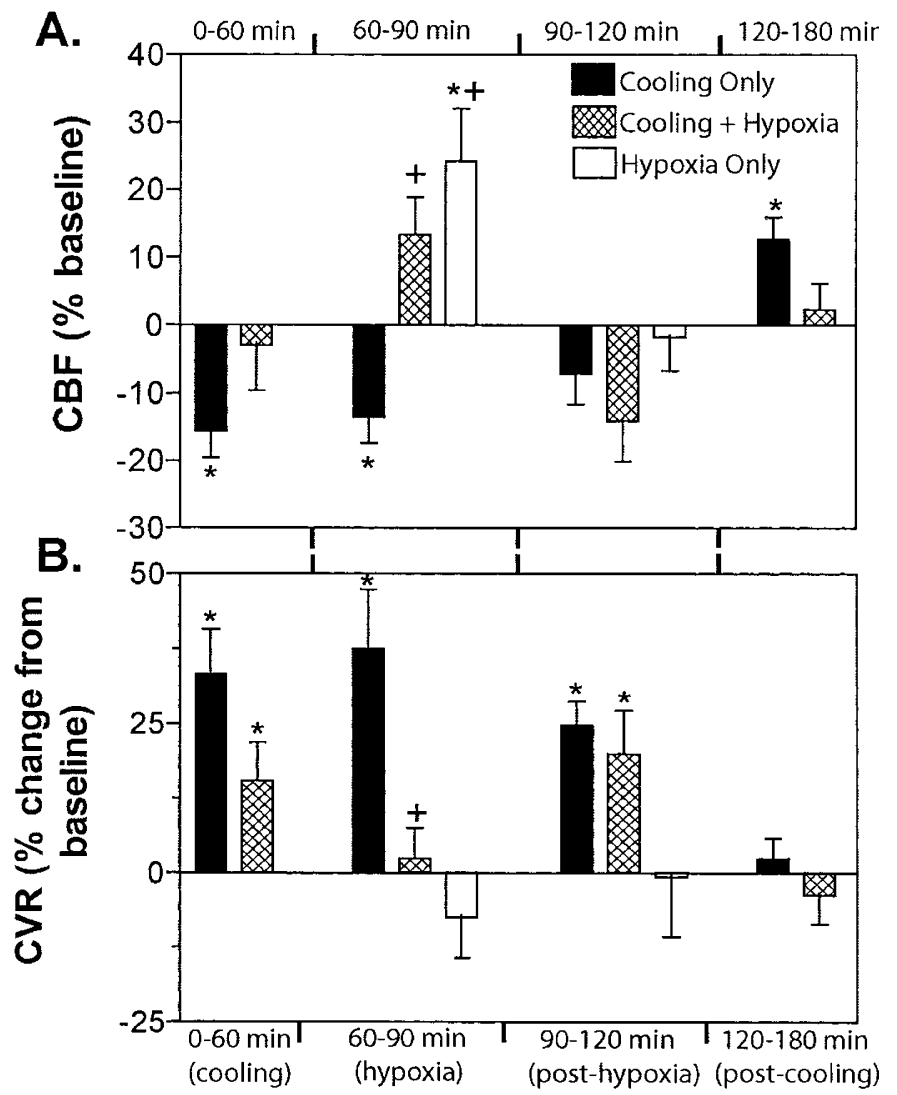

Figure 4. $A$, fetal cerebral blood flow $(C B F)$. Data shown under " $0-60 \mathrm{~min}$ " represent the first $60 \mathrm{~min}$ of cooling for the cooling control (solid bars, $n=7$ ) and cooling with hypoxia (cross-hatched bars, $n=6$ ) protocols. Data shown under "60-90 min" represent the 60- to 90-min period for the cooling control and cooling with hypoxia protocols, as well as the hypoxia controls (white bars, $n=7$ ). Data shown under " $90-120 \mathrm{~min}$ " represent the final $30 \mathrm{~min}$ of cooling for the cooling control and cooling with hypoxia protocols, and the 30 -min period after hypoxia for the hypoxia controls. Data shown under "120-180 min" represent the 1-h period after discontinuation of cooling in the cooling control and cooling with hypoxia protocols. $B$, fetal cerebral vascular resistance $(C V R)$. Legend same as for $A$. $\left(^{*}\right.$, significant difference from baseline period; + , significant difference from the cooling control protocol)

cooling period before hypoxia. Cerebral blood flow had not changed measurably at this time, whereas calculated resistance to flow averaged $16 \pm 6 \%$ above baseline. Blood pressure did not change significantly with administration of hypoxia, whereas cerebral blood flow increased to an average of $13 \pm$ $6 \%$ above baseline, a value significantly different from that of animals at the same time point in the cooling control experiments. The increase in cerebral blood flow during hypoxia and cooling was commensurate with a return of cerebral vascular resistance to baseline levels. Blood pressure remained unchanged during recovery from hypoxia while cooling was continued. Cerebral blood flow decreased to average $14 \pm 6 \%$ less than baseline at this time in association with continuing increased resistance to flow, averaging $22 \pm 6 \%$ above baseline. On cessation of cooling, blood pressure, flow, and calculated resistance to flow all returned to baseline levels.

In hypoxia control experiments, blood pressure rose significantly from $44 \pm 1 \mathrm{~mm} \mathrm{Hg}$ to $48 \pm 1 \mathrm{~mm} \mathrm{Hg}$ on initiation of hypoxia, and then returned to baseline levels during recovery

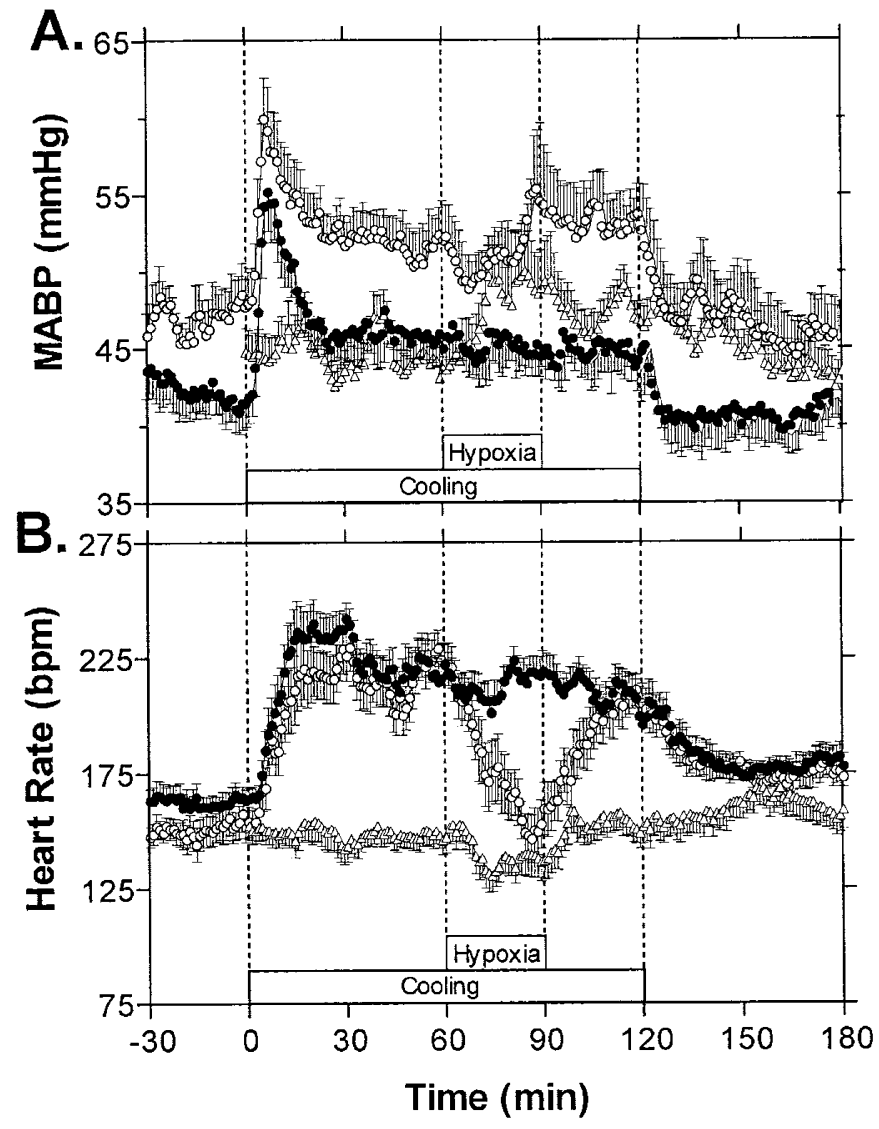

Figure 5. $A$, fetal mean arterial blood pressure $(M A B P)$ throughout the cooling control $(\bigcirc, n=13)$, cooling with hypoxia $(O, n=10)$, and hypoxia control $(\triangle, n=10)$ protocols. $B$, fetal heart rate throughout the cooling control $(\bigcirc, n=13)$, cooling with hypoxia $(\circ, n=10)$, and hypoxia control $(\triangle, n=$ 10) protocols.

(data not shown). On induction of hypoxia, cerebral blood flow increased significantly to average $24 \pm 8 \%$ above baseline and then returned to baseline levels during the recovery period. Resistance to flow did not change measurably during these experiments.

Oxygen extraction. Mean cerebral oxygen extraction is presented in Figure 6. With cooling alone there were no significant changes in oxygen extraction throughout the experiments. With hypoxia superimposed on cooling and with hypoxia alone, oxygen exaction was significantly increased during the hypoxic interval.

Heart rate. Changes in fetal heart rate during each of the protocols are shown in Figure 5B. With cooling a pronounced tachycardia was observed. With superimposed hypoxia heart rate gradually decreased throughout the hypoxic period, and then increased again during the recovery from hypoxia while cooling continued. After termination of cooling, heart rate returned to baseline levels. There was no significant difference between the baseline heart rate of the cooling with hypoxia and hypoxia control experiments. However, both of these protocols demonstrated a baseline heart rate that was significantly greater than that of the cooling control experiments $(p<0.01)$. 


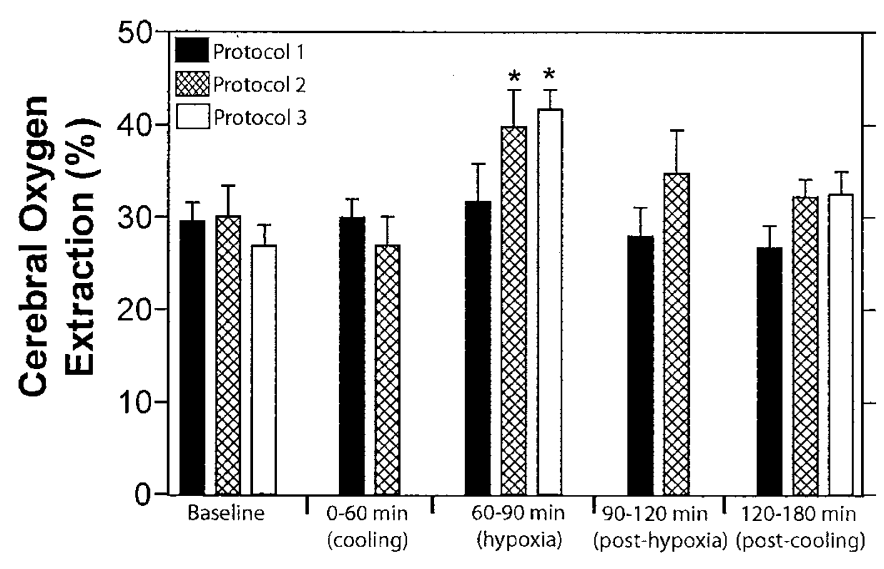

Figure 6. Fetal cerebral oxygen extraction. Data shown over "baseline" represent the baseline period for the cooling control (solid bars, $n=9$ ), cooling with hypoxia (cross-hatched bars, $n=6$ ), and hypoxia control protocol (white bars, $n=8$ ). Data shown over "0-60 min" represent extraction after 60 min of cooling for the cooling control and cooling with hypoxia protocols. Data shown over "60-90 min" represent the 60-90-min period for all three protocols. Data shown over "90-120 min" represent the final $30 \mathrm{~min}$ of cooling for the cooling control and cooling with hypoxia protocols. Data shown over "120-180 min" represent the 1-h period after discontinuation of cooling in the cooling control and cooling with hypoxia protocols, as well as the 1-h period after discontinuation of hypoxia in the hypoxia controls. (*, significant difference from baseline period)

\section{DISCUSSION}

The principal findings of the present study are that mild hypothermia (decrease in brain temperature of $1.6^{\circ} \pm 0.1^{\circ} \mathrm{C}$ ) in the ovine fetus results in significant decreases in cerebral oxygen consumption and cerebral blood flow, a significant increase in cerebral vascular resistance, and no change in oxygen extraction. Taken together these data provide evidence of effective coupling of cerebral metabolic rate and oxygen delivery in the fetal sheep during hypothermia. In addition, the findings of the present study demonstrate that fetal compensatory cardiovascular responses to hypoxia remain intact during mild hypothermia.

Effect of hypothermia on cerebral metabolic rate. Wholebody oxygen consumption increases during mild to moderate hypothermia owing to thermogenic responses (9). However, the oxygen consumption of most individual organs falls in response to hypothermia because of the biophysical effect of temperature on metabolic activity. The brain is no exception, as its oxygen consumption decreases 50 to $75 \%$ for every $10^{\circ} \mathrm{C}$ decrease in brain temperature $(1,4,13)$. In the present study, the effect of hypothermia on fetal cerebral oxygen consumption was similar to that of previous reports in the newborn and adult, with a $1.6^{\circ} \mathrm{C}$ decrease in brain temperature resulting in a $25 \%$ decrease in cerebral oxygen consumption. The effect of temperature on metabolic rate is often described by calculation of the $\mathrm{Q}_{10}$ coefficient; the ratio of two different rates of oxygen consumption at two temperatures separated by $10^{\circ} \mathrm{C} . \mathrm{Q}_{10}$ measurements in the brain have been reported to range between 2.2 and $4.6(13,14)$. The $\mathrm{Q}_{10}$ value is typically calculated by linear regression analysis of the relationship between temperature and oxygen consumption. Although extrapolation of the data from the present study results in a $\mathrm{Q}_{10}$ of approximately
3.8 , the range of temperatures over which cerebral metabolism was measured was not wide enough to permit a rigorous calculation of the $\mathrm{Q}_{10}$ value.

Coupling of fetal cerebral oxygen consumption and delivery. In the newborn and adult, lowering cerebral oxygen consumption with either hypothermia $(1,4,6)$ or barbiturates $(2,3,5)$ results in commensurate decreases in cerebral oxygen delivery, with little or no change in cerebral oxygen extraction. In the present study, we observed in the fetus a similar relationship between cerebral oxygen consumption and cerebral oxygen delivery (Fig. 6), such that oxygen extraction remained constant during cooling. This finding supports the previously suggested idea that, under normal conditions, the diffusion of oxygen is not a limiting factor in cerebral oxygen consumption despite the relatively small driving pressure for oxygen diffusion between the fetal capillaries and brain tissue (7). The present study also is in agreement with previous work showing that the fetus responds to increased arterial oxygen tensions by decreasing cerebral blood flow rather than increasing cerebral oxygen consumption as would be expected if cerebral oxygen consumption were normally limited by oxygen diffusion (15).

The possibility exists that the decrease in cerebral blood flow and oxygen consumption during cooling was a forced result of increased cerebrovascular resistance caused by the cold stimulus. However, this explanation appears unlikely inasmuch as an increase in cerebral oxygen extraction during cooling was not observed. As demonstrated when hypoxia was superimposed on cooling, the brain was still capable of increased oxygen extraction during cooling, ruling out the possibility that oxygen diffusion became a limiting factor during cooling.

Effect of hypothermia on fetal cardiovascular responses to hypoxic stress. The cardiovascular responses of the fetal sheep exposed to acute hypoxia include a rapid onset of bradycardia followed by a more delayed onset of increased arterial blood pressure and decreased peripheral blood flow. The increase in peripheral vascular resistance, combined with decreased resistance in the vital organs, allows blood flow to be redistributed to the heart, adrenals, and brain (7). Hypothermia did not appear to have a significant effect on cerebral blood flow responses to hypoxia. With superimposed hypoxia, cerebral blood flow increased and cerebrovascular resistance decreased to levels significantly different from those of cooling controls, and cerebral oxygen extraction increased significantly above baseline values.

In the fetal sheep, cold exposure results in a pronounced tachycardia mediated by increased plasma catecholamine levels (16). In contrast, acute moderate hypoxia results in an acute bradycardia as a result of a carotid chemoreflex (17), a mechanism that minimizes cardiac work during hypoxic stress. The hypoxic bradycardia is typically transient, returning to baseline levels as plasma catecholamine concentrations increase gradually during the first 10 to 20 min of hypoxia $(17,18)$. The pronounced fall in heart rate throughout the hypoxic period of the cooling and hypoxia experiments demonstrates not only the fact that chemoreceptor function remains intact during cooling but also that its vagally mediated effects on heart rate override the effect of increased plasma catecholamines. Thus, the data of the present study provide evidence that the ability of the 
fetus to minimize the cardiac workload in response to hypoxia is also intact during hypothermia.

As mentioned earlier, the increase in peripheral vascular resistance and arterial blood pressure during hypoxia is an important component of the increase in blood flow to the vital organs. During hypothermia, peripheral vascular resistance and arterial blood pressure are also increased by increases in plasma catecholamines released from the adrenal gland (16). In the present study, there was no significant change in blood pressure when hypoxia was administered together with cooling, suggesting the effects of the two interventions are not additive. The fact that arterial blood pressure during combined cooling and hypoxia remains elevated at or above levels observed during hypoxia alone, despite the pronounced bradycardia observed during hypoxia with cooling, suggests once again that fetal cardiovascular responses to hypoxia remain intact during hypothermia.

Effect of hypothermia on subsequent hypothermic and hypoxic stress. An unexpected finding of the present study was the difference between arterial blood pressures in the baseline periods of the cooling control and cooling with hypoxia experiments carried out 72 or more hours later. Although the magnitude of the increase in blood pressure during hypothermia was similar between the two protocols, the absolute blood pressure was higher throughout the cooling with hypoxia experiments compared with the cooling control experiments. The cause of these findings is not readily apparent from these studies. Speculations on possible causes of this finding include increased cortisol release during the first exposure to hypothermia, resulting in increased sensitivity of the vasculature to subsequent adrenergic stimulation. Alternatively, it may be simply a result of the normal maturational increase in arterial blood pressure that occurs throughout gestation.

Another indication of a possible preconditioning effect of hypothermia is found in the modest cerebral blood flow response to hypoxia in the hypoxia control experiments. In the present study, hypoxia resulted in an approximately $25 \%$ increase in cerebral blood flow compared with 40 to $50 \%$ increases of flow observed in similar aged fetuses using identical laser Doppler methods $(12,19)$. Thus, there are some indications of preconditioning effects of hypothermia on flow responses 3 to $6 \mathrm{~d}$ later, but further studies are clearly needed to explore such a phenomenon.

\section{CONCLUSIONS}

The present study confirms the findings of previous reports on the tight coupling of cerebral oxygen delivery and metabolic rate of the brain in the ovine fetus. Despite the relatively hypoxic environment of the fetus relative to the adult, cerebral oxygen delivery is still tightly coupled with cerebral oxygen consumption. The present data also demonstrate that key cardiovascular responses of the fetal sheep to hypoxic stress, such as elevated arterial blood pressure, decreased heart rate, decreased cerebral vascular resistance, and increased cerebral blood flow and oxygen extraction, remain intact during mild hypothermia. These findings have important implications for the safety of hypothermic therapies in the clinic in which any loss of compensatory responses to hypoxia might outweigh the benefit of hypothermic treatment.

Acknowledgments. The authors thank Shannon Bragg for expert technical assistance and Alistair J. Gunn for helpful criticisms on the manuscript.

\section{REFERENCES}

1. Rosomoff HL, Holaday DA 1954 Cerebral blood flow and cerebral oxygen consumption during hypothermia. Am J Physiol 179:85-90

2. Nordstrom $\mathrm{CH}$, Rehncrona S 1978 Reduction of cerebral blood flow and oxygen consumption with a combination of barbiturate anaesthesia and induced hypothermia in the rat. Acta Anaesthesiol Scand 22:7-12

3. Steen PA, Newberg L, Milde JH, Michenfelder JD 1983 Hypothermia and barbiturates: individual and combined effects on canine cerebral oxygen consumption. Anesthesiology 58:527-532

4. Walter B, Bauer R, Kuhnen G, Fritz H, Zwiener U 2000 Coupling of cerebral blood flow and oxygen metabolism in infant pigs during selective brain hypothermia. J Cereb Blood Flow Metab 20:1215-1224

5. Lafferty JJ, Keykhah MM, Shapiro HM, Van Horn K, Behar MG 1978 Cerebral hypometabolism obtained with deep pentobarbital anesthesia and hypothermia $\left(30^{\circ} \mathrm{C}\right)$. Anesthesiology 49:159-164

6. Busija DW, Leffler CW 1987 Hypothermia reduces cerebral metabolic rate and cerebral blood flow in newborn pigs. Am J Physiol 253:H869-H873

7. Jensen A, Garnier Y, Berger R 1999 Dynamics of fetal circulatory responses to hypoxia and asphyxia. Eur J Obstet Gynecol Reprod Biol 84:155-172

8. Gunn AJ, Gunn TR, de Haan HH, Williams CE, Gluckman PD 1997 Dramatic neuronal rescue with prolonged selective head cooling after ischemia in fetal lambs. J Clin Invest 99:248-256

9. Gunn AJ, Bennet L 2001 Is temperature important in delivery room resuscitation? Semin Neonatol 6:241-249

10. Battin MR, Dezoete JA, Gunn TR, Gluckman PD, Gunn AJ 2001 Neurodevelopmental outcome of infants treated with head cooling and mild hypothermia after perinatal asphyxia. Pediatrics 107:480-484

11. Sawa R, Asakura H, Power GG 1991 Changes in plasma adenosine during simulated birth of fetal sheep. J Appl Physiol 70:1524-1528

12. Lan J, Hunter CJ, Murata T, Power GG 2000 Adaptation of laser-Doppler flowmetry to measure cerebral blood flow in the fetal sheep. J Appl Physiol 89:1065-1071

13. van der Linden J, Priddy R, Ekroth R, Lincoln C, Pugsley W, Scallan M, Tyden H 1991 Cerebral perfusion and metabolism during profound hypothermia in children: a study of middle cerebral artery ultrasonic variables and cerebral extraction of oxygen. J Thorac Cardiovasc Surg 102:103-114

14. Michenfelder JD, Theye RA 1968 Hypothermia: effect on canine brain and wholebody metabolism. Anesthesiology 29:1107-1112

15. Gleason CA, Jones Jr MD, Traystman RJ, Notter RH 1988 Fetal cerebral responses to ventilation and oxygenation in utero. Am J Physiol 255:R1049-R1054

16. Gunn TR, Johnston BM, Iwamoto HS, Fraser M, Nicholls MG, Gluckman PD 1985 Haemodynamic and catecholamine responses to hypothermia in the fetal sheep in utero. J Dev Physiol 7:241-249

17. Giussani DA, Spencer JA, Moore PJ, Bennet L, Hanson MA 1993 Afferent and efferent components of the cardiovascular reflex responses to acute hypoxia in term fetal sheep. J Physiol (Lond) 461:431-449

18. Iwamoto HS, Rudolph AM, Mirkin BL, Keil LC 1983 Circulatory and humoral responses of sympathectomized fetal sheep to hypoxemia. Am J Physiol 245:H767$\mathrm{H} 772$

19. Blood AB, Hunter CJ, Power GG 2002 The role of adenosine in regulation of cerebral blood flow during hypoxia in the near-term fetal sheep. J Physiol (Lond) 543:10151023 